\title{
Hop Domination in Graphs-II
}

\author{
C. Natarajan and S.K. Ayyaswamy
}

\begin{abstract}
Let $G=(V, E)$ be a graph. A set $S \subset V(G)$ is a hop dominating set of $G$ if for every $v \in V-S$, there exists $u \in S$ such that $d(u, v)=2$. The minimum cardinality of a hop dominating set of $G$ is called a hop domination number of $G$ and is denoted by $\gamma_{h}(G)$. In this paper we characterize the family of trees and unicyclic graphs for which $\gamma_{h}(G)=\gamma_{t}(G)$ and $\gamma_{h}(G)=\gamma_{c}(G)$ where $\gamma_{t}(G)$ and $\gamma_{c}(G)$ are the total domination and connected domination numbers of $G$ respectively. We then present the strong equality of hop domination and hop independent domination numbers for trees. Hop domination numbers of shadow graph and mycielskian graph of graph are also discussed.
\end{abstract}

\section{Introduction}

Domination in graphs is one of the fastest growing areas in Graph theory. Many authors contribute several interesting domination parameters to nurture the growth of this research area. An excellent treatment of several topics in domination can be found in two books $[4,5]$ written by Haynes et al. The following are some basic definitions and results to discuss further.

Key Words: Hop domination number, total domination number, connected domination number

2010 Mathematics Subject Classification: Primary 05C69; Secondary 05C75.

Received: November, 2013.

Revised: November, 2013.

Accepted: November, 2013. 
By an $n t c$ graph $G$ we mean a non trivial connected graph $G=(V, E)$ where $V$ is the set of vertices and $E$ is the set of edges of $G$. The distance between two vertices $u$ and $v$ of a graph $G$ is the length of the shortest path joining $u$ and $v$ in $G$ and is denoted by $d(u, v)$. A graph $G$ is said to be unicyclic if it has exactly one cycle. A double star is a tree obtained by joining the centers of two stars $K_{1, r}$ and $K_{1, s}$. We denote a double star of order $r+s+2$ by $D(r, s)$. A set $D \subset V$ is a dominating set of $G$ if every vertex $v \in V-D$ is adjacent to some vertex in $D$. A dominating set $D$ is said to be minimal if no subset of $D$ is a dominating set of $G$. The minimum cardinality of a minimal dominating set of $G$ is called the domination number of $G$ and is denoted by $\gamma(G)$. The upper domination number $\Gamma(G)$, is the maximum cardinality of a minimal dominating set of $G$. A set $D \subset V$ is a total dominating set of $G$ if every vertex $v \in V$ is dominated by at least one vertex in $D$. The minimum cardinality of such a set is called the total domination number of $G$ and is denoted by $\gamma_{t}(G)$. A dominating set $D$ is a connected dominating set of $G$ if the subgraph $\langle D>$ induced by $D$, is connected. The minimum cardinality of a connected dominating set of $G$ is called the connected domination number of $G$ which we denote by $\gamma_{c}(G)$. S.K. Ayyaswamy et al. [1] have recently defined a new domination parameter called hop domination number of a graph. The definition is as follows: A set $S \subset V$ of a graph $G$ is a hop dominating set(hdset, in short) of $G$ if for every $v \in V-S$, there exists $u \in S$ such that $d(u, v)=2$. The minimum cardinality of a hd-set of $G$ is called the hop domination number and is denoted by $\gamma_{h}(G)$. We present the hop domination number of a few well known graphs in the following proposition.

Proposition 1. ([1])

(i) For a complete graph $K_{n}, \gamma_{h}\left(K_{n}\right)=n$.

(ii)For a complete bipartite graph $K_{m, n}, \gamma_{h}\left(K_{m, n}\right)=2$.

(iii) For a path $P_{n}$ on $n$ vertices $\gamma_{h}\left(P_{n}\right)= \begin{cases}2 r, & \text { if } n=6 r ; \\ 2 r+1, & \text { if } n=6 r+1 ; \\ 2 r+2, & \text { if } n=6 r+s ; 2 \leq s \leq 5 .\end{cases}$

(iv) For a cycle $C_{n}$ of length $n, \gamma_{h}\left(C_{n}\right)= \begin{cases}2 r, & \text { if } n=6 r ; \\ 2 r+1, & \text { if } n=6 r+1 ; \\ 2 r+2, & \text { if } n=6 r+s ; 2 \leq s \leq 5 .\end{cases}$

(v) $\gamma_{h}\left(W_{n}\right)=3$ where $W_{n}$ is a wheel with $n-1$ spokes. 
(vi) $\gamma_{h}(P)=2$ where $P$ denotes the Petersen graph.

Proposition 2. [1] For every traingle-free graph $G$ without isolated vertices, $\gamma_{h}(G) \leq \gamma_{t}(G)$.

\section{Main Results}

It is in the usual practice to study the relation between the new domination parameter and the existing one. Likewise we study the equality of hop domination number and other domination parameters such as connected domination number and total domination number.

\subsection{Characterization of graphs with $\gamma_{h}=\gamma_{t}$}

We now construct a family of $\mathcal{T}$ trees having $\gamma_{h}=\gamma_{t}$ in the following. We define a family $\mathcal{T}$ of trees $T$ that can be obtained form the disjoint union of $k \geq 1$ double stars. Let $C$ be the set of central vertices of the double stars. Add $k-1$ edges between the vertices of $V-C$ so that the resulting graph is a tree.

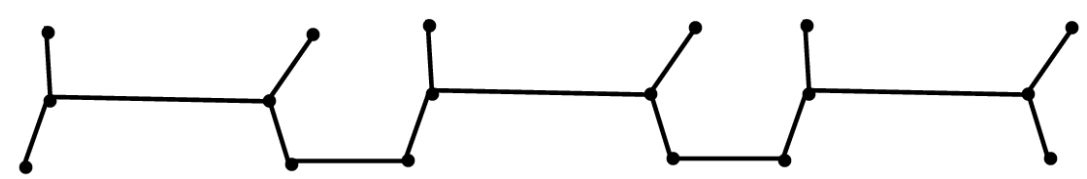

Figure 1: Tree $T$ in $\mathcal{T}$

Theorem 3. For any tree $T, \gamma_{h}(T)=\gamma_{t}(T)$ if and only if $T \in \mathcal{T}$.

Proof. Assume that $\gamma_{h}(T)=\gamma_{t}(T)$.

Let $D$ be a total dominating set. By Proposition $2 D$ is a hop dominating set as well. Choose a vertex $a_{1}$ which is support vertex of leaves only. This is possible since $T$ is a tree. If $a_{1} \in P_{l} \subset D$ where $\left.P_{l}=<a_{1}, b_{1}, \ldots, x_{l}\right\rangle$ is a path, then $l=2$. Suppose $l \geq 3$. Then $c_{1}$ hop dominates $a_{1}$ and all those vertices hop dominated by $a_{1}$. Therefore, $D-\left\{a_{1}\right\}$ is a hop dominating set 
and so $\gamma_{h}(T) \leq|D|-1<\gamma_{t}(T)$, a contradiction. Thus $P_{l}=<a_{1}, b_{1}>$. Let $a_{2} \in D$ be such that $d\left(a_{2}, b_{1}\right)$ is minimum. As $D$ is a hop dominating set also, we have $d\left(a_{2}, b_{1}\right)=2$ or 3 . We claim that $d\left(a_{2}, b_{1}\right)=3$. Suppose $b_{1} v a_{2}$ is a path in $T$. Then there exists $b_{2} \in N\left(a_{2}\right) \cap D$, as $D$ is a total dominating set.

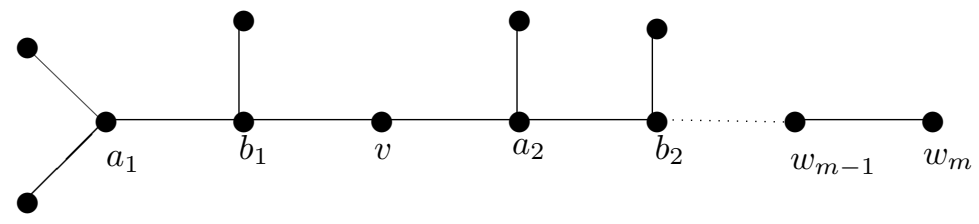

Figure 2:

If $b_{2}$ is a support vertex of leaves only, then $D-\left\{a_{1}, b_{2}\right\} \cup\{v\}$ is a hop dominating set since $v$ hop dominates $b_{2}$ and all those vertices hop dominated by $a_{1}$ and $b_{2}$. This implies that $\gamma_{h}(T)<\gamma_{t}(T)$, a contradiction. If $b_{2}$ is not a support of leaves only, let $P_{m}=<b_{2}=w_{1}, w_{2}, \ldots, w_{m}>\subset D$ be a path, then $w_{m-1}$ is in $D$ whereas $w_{m-1}$ is not needed to hop dominate any vertex of $T$ and hence $D-\left\{a_{1}, w_{m-1}\right\} \cup\{v\}$ is a hop dominating set and consequently $\gamma_{h}(T)<\gamma_{t}(T)$, a contradiction. Thus $d\left(b_{1}, a_{2}\right)=3$. Now, let $a_{2} \in P_{s} \subset D$ where $P_{s}=<a_{2}=u_{1}, u_{2}, \ldots, u_{s}>, s \geq 3$, then as proved earlier we can show that $D-\left\{a_{2}\right\}$ is a hop dominating set which leads to a contradiction to our assumption that $\gamma_{h}(T)=\gamma_{t}(T)$. Proceeding like this, we get $D=\cup\left\{a_{i}, b_{i}\right\}$ and $d\left(b_{i}, a_{i+1}\right)=3$. This shows that $T \in \mathcal{T}$.

We define a family of unicyclic graphs $\mathcal{T}^{*}$ as follows:

$\mathcal{T}^{*}=\{T \cup e: T \in \mathcal{T}, e$ is an edge joining any two leaves or a leaf and an internal vertex which is not a support vertex in $T$.

Theorem 4. Let $G$ be a unicyclic graph with the unique cycle $C$. Then $\gamma_{h}(G)=\gamma_{t}(G)$ if and only if $G$ is $C_{4}$ or $G \in \mathcal{T}^{*}$.

Proof. Let $\gamma_{h}(G)=\gamma_{t}(G)$.

If $G$ is just a cycle $C$, then $\gamma_{h}(C)=\gamma_{t}(C)$ if and only if $G=C_{4}$. So we assume 
that $G$ is not a mere cycle. Then $G$ has at least one pendant vertex. Let $D$ be a total dominating set of $G$ and let $P=<a_{0}, a_{1}, \ldots, a_{m}>$ be a longest path in $G$. If $a_{1} \in P_{l} \subset D$, then as discussed in Theorem $3, l=2$. That is, $P_{l}=<a_{1}, b_{1}>\subset D$ and $\left(N\left(b_{1}\right)-\left\{a_{1}\right\}\right) \cap D=\emptyset$ and $\left(N\left(a_{1}\right)-\left\{b_{1}\right\}\right) \cap D=\emptyset$. Let $a_{2}, b_{2} \in D$ be such that $a_{2}$ is nearest to $b_{1}$. Then $d\left(b_{1}, a_{2}\right) \leq 3$. We claim that $d\left(b_{1}, a_{2}\right)=3$. Suppose $d\left(b_{1}, a_{2}\right)=2$ and consider the path $b_{1} v a_{2}$ in $D$.

Case 1: There is a path $P=\left\{b_{2}=w_{1}, w_{2}, \ldots, w_{m}\right\}$ from $b_{2}$ such that $b_{2} \in C$, $a_{2} \notin P$ and $P \cap C=\left\{b_{2}\right\}$ only where $C$ is the unique cycle in $G$. Then as discussed in Theorem $3, w_{m-1} \in D$ whereas $w_{m-1}$ is not in any hd-set so that $D-\left\{a_{1}, w_{m-1}\right\} \cup\{v\}$ is hd-set which contradicts our assumption that $\gamma_{h}(G)=\gamma_{t}(G)$.

Case 2: Let $b_{2} \in C$ and there is no path from $b_{2}$. If the cycle $C$ does not contain $a_{2}$, then clearly $D-\left\{a_{1}, a_{2}\right\} \cup\{v\}$ is a hd-set. So, assume that $C$ contains $a_{2}$ and $b_{2}$. Let $C$ be $<b_{2}, a_{2}, w_{1}, w_{2}, \ldots, w_{m-1}, w_{m}=b_{2}>$. We shall assume that $\operatorname{deg}\left(w_{i}\right)=2$ for all $i=1,2, \ldots, m$; otherwise as discussed in Case 1 we can arrive at a contradiction. If $w_{m-2} \in D$, then $w_{m-2}$ hop dominates $b_{2}$ and $a_{2}$ hop dominates the neighbours of $b_{2}$. So $D-\left\{a_{1}, b_{2}\right\} \cup\{v\}$ is a hd-set of $G$. If $w_{m-1} \in D$, then $w_{m-1}$ hop dominates $a_{2}$ and $v$ hop dominates all neighbours of $a_{2}$. So $D-\left\{a_{1}, a_{2}\right\} \cup\{v\}$ is a hd-set. If $w_{m-3} \in D$, then $w_{m-3}$ hop dominates $w_{m-1}$ and $v$ hop dominates $b_{2}$. So $D-\left\{a_{1}, b_{2}\right\} \cup\{v\}$ is a hd-set. All these cases imply $\gamma_{h}(G)<\gamma_{t}(G)$. Thus $d\left(b_{1}, a_{2}\right)=3$. If $a_{2} b_{2} c_{2}$ is a path in $D$, then $D-\left\{a_{2}\right\}$ is a hd-set since $a_{2}$ is hop dominated by $c_{2}$ and all neighbours of $a_{2}$ are hop dominated by $b_{2}$. Thus $a_{2} \in P_{l} \subset D$ implies $l=2$, that is, neither the neighbours of $a_{2}$ nor the neighbours of $b_{2}$ other than $a_{2}$ and $b_{2}$ are in $D$. Proceeding like this we get $D=\cup\left\{a_{i}, b_{i}\right\}$ such that $a_{i}, b_{i}$ forms $K_{2}$ and $d\left(b_{i+1}, a_{i}\right)=3$. Again, as $D=\cup\left\{a_{i}, b_{i}\right\}$, the vertices $a_{i} \mathrm{~s}$ and $b_{i}$ s are the only supports of leaves in $G$. This together with the fact that $d\left(b_{i}, a_{i+1}\right)=3$ implies $G \in \mathcal{T}^{*}$.

\subsection{Characterization of a family of graphs for which $\gamma_{h}(G)=\gamma_{c}(G)$}

Theorem 5. Let $T$ be a tree of order $n>3$. Then $\gamma_{h}(T)=\gamma_{c}(T)$ if and only if $T$ is a double star.

Proof. Assume that $\gamma_{h}(T)=\gamma_{c}(T)$.

Let $l$ denote the number of leaves in $T$. We first show that every internal 
vertex of $T$ is a support vertex.

Suppose $T$ has internal vertices which are support vertices. Choose one such vertex, say $v$ such that $N(v)$ contains a support vertex $u$. Since $T$ has no cycle, there exists $w \in N(v)$ such that $d(u, w)=2$. Then $v$ hop dominates all the leaves at $u$ and $w$ hop dominates $u$. Therefore we can choose a hop dominating set of $T$ not containing $u$ and its leaves so that $\gamma_{h}(G) \leq n-l-1<$ $\gamma_{c}(G)$, a contradiction. Hence every internal vertex in $T$ is a support vertex.

We next prove that $T$ has exactly two support vertices. Suppose $T$ has more than two support vertices. Let $P: v_{1}, v_{2}, \cdots v_{n}$. Then $v_{1}$ and $v_{i}$ are leaves and all other $v_{i}$ s are support vertices in $T$.

Clearly, $v_{2}$ is hop dominated by $v_{4}$ and the leaves of $v_{2}$ including $v_{1}$ are hop dominated by $v_{3}$. Hence we can find a hop dominating set $S$ with $S \leq$ $n-l-1<n-l=\gamma_{c}$

We construct a family $\mathcal{G}$ of unicyclic graphs as follows:

Operation $U_{1}$ : Join any two non-adjacent vertices of a double star $D(r, s)$ where $r, s \geq 2$.

Operation $U_{2}$ : If $r=1$, then we have a double star $\left(K_{1,1}, K_{1, s}\right)$. In this case we join any two non-adjacent vertices except $v$ and $u_{1}$.

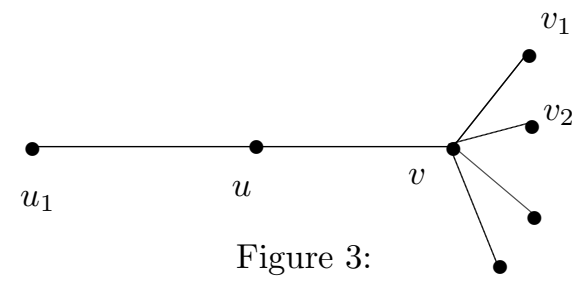

$v_{s}$ 
Theorem 6. Let $G$ be a unicyclic graph. Then $\gamma_{h}(G)=\gamma_{c}(G)$ if and only if $G \in \mathcal{G}$.

Proof. Assume that $\gamma_{h}(G)=\gamma_{c}(G)$. Let $C$ be the cycle in $G$ of length $m$ and let $X$ denote the set of all vertices of degree 2 in $C$. Let $t$ denote the number of trees in $G$ of diameter $\geq 2$ from a vertex in $C$ and $l$ be the number of leaves in $G$.

We first show that $m \leq 4$.

Case 1: $m=6 r_{1}+s_{1} ; r_{1}>0$ and $0 \leq s_{1} \leq 5$.

Case 1.1: Let $m=6 r_{1}$.

If $u=u_{1} u_{2} \ldots u_{x}$ is a longest path of length $\geq 2$ in $G-C$ from $u \in C$, then we can find a hop dominating set $S$ not containing $u_{x-1}$ and $u_{x}$. As $m \geq 4$, this $S$ can be chosen such that it does not contain any leaves of $G$. Also we know that $\gamma_{h}(C)=2 r_{1}$. Therefore, we have $\gamma_{h}(G) \leq$ $2 r_{1}+n-m-t-l$.

i.e., $\leq 2 r_{1}+n-6 r_{1}-t-l<n-l-2 \leq \gamma_{c}(G)$.

Case 1.2: Let $m=6 r_{1}+1$.

In this case we have $\gamma_{h}(G) \leq 2 r_{1}+1+n-m-t-l$.

i.e., $\leq n-l-\left(4 r_{1}+2\right)<n-l-2 \leq \gamma_{c}(G)$.

Case 1.3: Let $m=6 r_{1}+a ; 2 \leq a \leq 5$.

Then $\gamma_{h}(G) \leq 2 r_{1}+2+n-m-l-t$.

i.e., $\leq n-l-\left(4 r_{1}+t+a-2\right)<n-l-2 \leq \gamma_{c}(G)$.

Case 2: Let $m=5$.

Then $\gamma_{h}(G) \leq 2+\gamma_{h}(G-C)<3+\gamma_{c}(G-C)=\gamma_{c}(G)$, a contradiction.

Therefore, if $\gamma_{h}(G)=\gamma_{c}(G)$, then $m \leq 4$.

Case 3: Let $m=3$.

Case 3.1: Let $m=3$ and $|X|=1$.

Let $u, v \in C-X$ and $w \in X$.

We claim that $u$ and $v$ can be supports of leaves only.

If not, let there be a longest path $u=u_{1} u_{2} \ldots u_{y} ; y \geq 3$. Then we can find a hop dominating set not containing $u_{y-1}$ and $u_{y}$. But the unique $\gamma_{c}$-set of $G$ contains all $u_{i}$ s and $v$ and so $\gamma_{c}(G)>\gamma_{h}(G)$, a contradiction. Therefore, the $\gamma_{h}$-set of $G$ will consist of $u$ and one of the leaves of $u$ or 
$v$ and one of the leaves of $v$. This implies $\gamma_{h}(G)=2$. As $\{u, v\}$ is the $\gamma_{c}$-set of $G$, we have $\gamma_{h}(G)=\gamma_{c}(G)$.

Case 3.2: Let $m=3$ and $|X|=2$.

Let $u \in V(C)-X$ and $v, w \in X$.

Claim: $u$ is a support of a tree in $G-C$ with diameter 2 .

Suppose $u$ supports a tree in $G-C$ with diameter $i 2$. Let $u=u_{1} u_{2} \ldots u_{t}$ be the longest path in $G$. Then we can form a hop dominating set not containing $u_{t-1}$ and $u_{t}$; but the unique $\gamma_{c}$-set of $G$ contains $u=$ $u_{1} u_{2} \ldots u_{t-1}$. Thus $\gamma_{h}(G)<\gamma_{c}(G)$, a contradiction.

We next observe that $u$ cannot be a support of leaves alone, since, in that case $\gamma_{c}(G)=1$ as $\{u\}$ is the $\gamma_{c}$-set of $G$ whereas a $\gamma_{h}$-set of $G$ contains two vertices, namely $u$ and one of its leaves.

Case 3.3: Let $m=3$ and $|X|=0$.

This case is not possible, since $\gamma_{h}(G)<3+r$ whereas $\gamma_{c}(G)=3+r$, where $r$ is the number of internal vertices in $G-C$.

Case 4: Let $m=4$.

We claim that $2 \leq|X| \leq 4$.

Suppose $|X|=0$.

If $G$ has $r$ number of internal vertices other than $V(C)$, then $\gamma_{h}(G) \leq 2+r$ whereas $\gamma_{c}(G)=4+r$, a contradiction.

Similarly, we can show that $|X| \neq 1$.

The graph $G$ for $|X|=2$ is $G_{1}$.

The graph $G$ for $|X|=3$ is $G_{2}$.

The graph $G$ for $|X|=4$ is $C_{4}$.

The converse is trivial. 


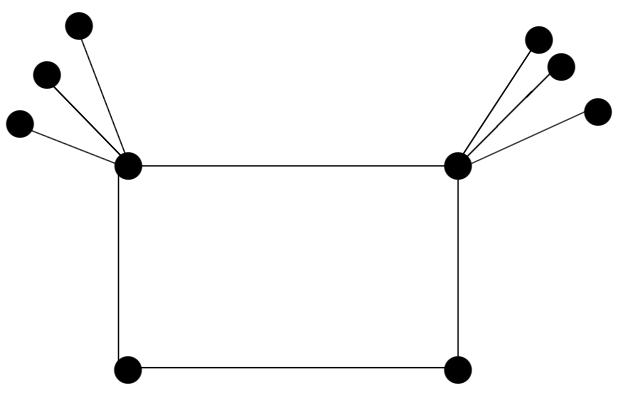

Figure 4: $G_{1}$

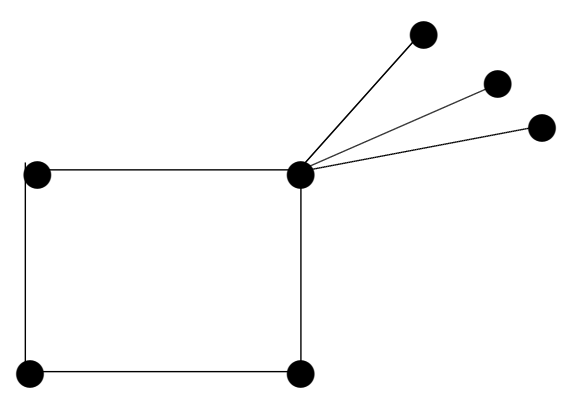

Figure 5: $G_{2}$

\subsection{Strong equality of hop domination and hop independent dom-} ination numbers

In [6], Haynes et al. obtained strong equality of domination number and other domination parameters for trees. Similarly we have the following strong equality of hop domination and hop independent domination numbers for trees.

Definition 7. For a graph $G$, we say that $\gamma_{h}(G)$ strongly equals $\gamma_{h i}(G)$, if $\gamma_{h}(G)=\gamma_{h i}(G)$ and every $\gamma_{h}$-set of $G$ is a $\gamma_{h i}$-set of $G$. The strong equality of these two sets is denoted by $\gamma_{h}(G) \equiv \gamma_{h i}(G)$.

Proposition 8. Let $T$ be a tree of diameter $\leq 5$. Then $\gamma_{h}(T) \equiv \gamma_{h i}(T)$.

Proof. Let $P$ be a longest path in $T$ of length $d$. Then $d \leq 5$. Case 1 Let 
$d=5$. Then $P$ has the vertices $v_{1}, v_{2}, v_{3}, v_{4}, v_{5}$ and $v_{6}$. Clearly $\left\{v_{3}, v_{4}\right\}$ is the only $\gamma_{h}$-set of $P$ which is also a $\gamma_{h i}$-set of $P$.

Case 2 Let $d=4$. Then $P=<v_{1}, v_{2}, v_{3}, v_{4}, v_{5}>$. Clearly $\left\{v_{3}, v_{4}\right\}$ is the only $\gamma_{h}$-set of $T$ which is also an h-independent set of $T$.

Remark: The converse of the above proposition is not true. For example, consider the following tree $T$.

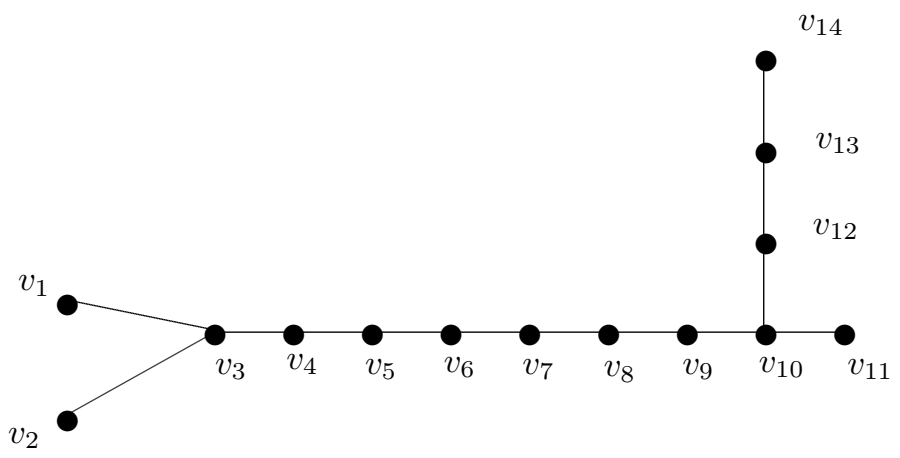

Figure 6: $T$

For this tree $T \gamma_{h}(T) \equiv \gamma_{h i}(T)$ since $\left\{v_{4}, v_{5}, v_{10}, v_{12}\right\}$ is the only set which is hop dominating as well as hop indepedent dominating set. But $\operatorname{diam}(T)>5$.

Proposition 9. Let $P$ be a path with $n \geq 4$ vertices. Then $\gamma_{h}(P) \equiv \gamma_{h i}(P)$ if and only if $n \equiv 0,4,5(\bmod 6)$.

Proof. Let $V(P)=\left\{v_{1}, v_{2}, \cdots, v_{n}\right\}$. Then every $\gamma_{h}$-set $S$ of $T$ contains the pairs of vertices $v_{3}, v_{4} ; v_{9}, v_{10} ; \cdots$, in general $v_{6 t-3}, v_{6 t-2}$. Suppose $n=$ $6 t+3$. Then $v_{6 t+2}$ can be hop dominated by $v_{6 t}$. But $v_{6 t-2}$ is already in the set $S$ which is of distance 2 from $v_{6 t}$. Therefore the $\gamma_{h}$-set $S=$ $\left\{v_{3}, v_{4}, v_{9}, v_{10}, \cdots, v_{6 t-3}, v_{6 t-2}, v_{6 t}\right\}$ will not be h-independent. This is the case for $n=6 t+1$ and $n=6 t+2$. Therefore, $n=6 t+4$ and $n=6 t+5$ is same as writing $n \equiv 0,4,5(\bmod 6)$. 
Definition 10. A caterpillar $T$ is full footed (FFC) if every internal vertex is a support vertex of at least one leaf. We say that a central path in a FFC is of order $n$ if FFC has $n$ number of internal vertices.

Theorem 11. An FFC T of order $n$ has the property $\gamma_{h}(T) \equiv \gamma_{h i}(T)$ if and only if $n=4 t$.

Proof. Let $\left\{v_{1}, v_{2}, \cdots, v_{n}\right\}$ be the set of internal vertices of $T$. If $n=4 t$, then the only $\gamma_{h}$-set is $\left\{v_{2}, v_{3}, v_{6}, v_{10}, \cdots, v_{4 t-2}, v_{4 t-1}\right\}$ where $v_{4 t-1}$ is needed to hop dominate the leaves of $v_{4 t}$. Therefore $\gamma_{h}(T) \equiv \gamma_{h i}(T)$. If $n \neq 4 t$, for instance $n=4 t-1$, then the set $S=\left\{v_{2}, v_{3}, v_{6}, v_{7}, \cdots, v_{4 t-6}, v_{4 t-5}, v_{4 t-3}, v_{4 t-2}\right\}$ is a $\gamma_{h}$-set of $T$ with $d_{G}\left(v_{4 t-5}, v_{4 t-3}\right)=2$ and so $S$ is not a $\gamma_{h i}$-set of $T$ implying $\gamma_{h}(T)$ is not strongly equal to $\gamma_{h i}(T)$. Similarly, if $n=4 t+1$, then we can find a $\gamma_{h}$-set containing the leaves $v_{4 t-1}$ and $v_{4 t-3}$ which are of distance 2. Other cases $n=4 t+2$ and $n=4 t+3$ can be argued similarly.

\subsection{Hop domination number of shadow graph and mycielskian graph of a graph}

Theorem 12. For any graph $G, \gamma_{h}(\operatorname{Sh}(G))=\gamma_{h}(G)$.

Proof. Let $D$ be a hop dominating set of $G$. Let $v^{\prime}$ be the twin of a vertex $v \in$ $G$. If $v \in D$, then $d\left(v, v^{\prime}\right)=2$ and hence $v$ hop dominates $v^{\prime}$. Otherwise, let $u \in D$ hop dominates $v$. Then there exists $w \in G$ such that $w \in N(u) \cap N(v)$. Now, $w \in N(v)$ implies $w$ and $v^{\prime}$ are adjacent. This together with $d(u, w)=1$ implies $v^{\prime}$ is hop dominated by $u$. This proves that $\gamma_{h}(\operatorname{Sh}(G))=\gamma_{h}(G)$.

Corollary 13. For any graph $G, \gamma_{h}(\mu(G))=\gamma_{h}(G)$.

Proof. Let $D$ be a hop dominating set of $G$. As $\mu(G)=S h(G) \cup\{c\}$ where $c$ is a vertex adjacent to all the twins of $v \in V(G)$, any vertex in $D$ hop dominates c. Thus $\gamma_{h}(\mu(G))=\gamma_{h}(G)$.

\section{Open Problems}

We conclude this study on hop domination with the following open problems which lead to further research in this topic. 
1. Characterize the family of graphs for which

(i) $\gamma_{h}(G)=\gamma_{h i}(G)$

(ii) $\Gamma_{h}(G)=\beta_{h}(G)$

(iii) $\gamma_{h}(G)=\gamma_{c}(G)$ (other than trees and unicyclic graphs)

(iv) $\gamma_{h}(G)=\gamma_{t}(G)$ (other than trees and unicyclic graphs)

2. Determine complexity of these problems mentioned in 1 .

3. Characterize connected graphs $G$ with $\gamma_{h}(G)=n-1$.

Acknowledgments: We thank Prof. M.Chellali and anonymous Referees for their helpful suggestions.

\section{References}

[1] S.K. Ayyaswamy and C. Natarajan, Hop domination in graphs, submitted to Discussiones Mathematicae Graph Theory.

[2] G. Chartrand and L. Lesniak, Graphs and Digraphs, Chapman and Hall, CRC, $4^{\text {th }}$ edition, 2005.

[3] M. Chellali, O. Favaron, A. Hansberg and L. Volkmann, k-domination and k-independence in graphs: A survey, Graphs and Combinatorics, 2012.

[4] T.W. Haynes, S.T. Hedetniemi and P.J. Slater, Fundamentals of Domination in graphs, Marcel Dekker, New York, 1998.

[5] T.W. Haynes, S.T. Hedetniemi and P.J. Slater, Domination in Graphs Advanced Topics, Marcel Dekker Inc., 1998.

[6] T.W. Haynes, M.A. Henning and P.J. Slater, Strong equality of domination parameters in trees, Discrete Mathematics, 260:77-87,2003.

[7] J.R. Lewis, Vertex-edge and Edge-vertex paramaters in graphs, $\mathrm{PhD}$ Thesis, Graduate School of Clemson University, August 2007.

[8] N. Sridharan, V.S.A. Subramanian and M.D. Elias, Bounds on the distance two-domination number of a graph, Graphs and Combinatorics, 18:667-675, 2002. 
C. Natarajan,

Department of Mathematics,

School of Humanities and Sciences,

SASTRA University,

Thanjavur - 613 401, Tamilnadu, India.

Email: natarajan_c@maths.sastra.edu

S.K. Ayyaswamy,

Department of Mathematics,

School of Humanities and Sciences,

SASTRA University,

Thanjavur - 613 401, Tamilnadu, India.

Email: sjcayya@yahoo.co.in 